\title{
Comparative Study of the Effect of Clonidine and Butorphanol as Adjuvant to Intrathecal Bupivacaine for Post Operative Analgesia in Lower Abdominal and Lower Limb Surgeries
}

\author{
Rumani Ruku', Sanam Kassana ${ }^{2}$ \\ ${ }^{1,2}$ Department of Anaesthesiology and Intensive Care, Govt. Medical College, Jammu \\ Corresponding Author: Rumani Ruku
}

DOI: https://doi.org/10.52403/ijrr.20220135

\begin{abstract}
Background: Intrathecal adjuvants to local anesthetic enhance the quality and duration of spinal anaesthesia. The present study was aimed to comparatively evaluate clonidine and butorphanol as adjuvants to intrathecal hyperbaric bupivacaine for subarachnoid blockade during lower abdominal and lower limb surgeries.

Patients and Method: Sixty adult consented patients, undergoing elective lower-abdominal and lower-limb surgeries under subarachnoid blockade, were inducted in a blind randomized prospective study conducted in the Department of Anesthesia, GMC Jammu and allocated into 2 equal groups to receive either $3.2 \mathrm{ml}$ of $0.5 \%$ hyperbaric bupivacaine $(16 \mathrm{mg})$ with $0.2 \mathrm{ml}$ of Clonidine $30 \mu \mathrm{gm}$ (Group I) or with $0.2 \mathrm{ml}$ of Butorphanol $200 \mu \mathrm{gm}$ (Group II). Patients were compared for the onset and duration of sensory and motor blockade, intraoperative hemodynamic changes, time to first rescue analgesia, etc.

Result: The mean onset time of sensory block was $3.18 \pm 0.56 \mathrm{~min}$ in Group I and 3.69 \pm 0.83 min in Group II. Complete motor block was achieved in $11.53 \pm 3.05 \mathrm{~min}$ in Group I and in $12.72 \pm 3.17 \mathrm{~min}$ in Group II, with no significant difference. $(\mathrm{p}=0.138)$ The duration of motor block was 294.28 $\pm 33.85 \mathrm{~min}$ in Group I, as compared to patients of butorphanol group $245.71 \pm 30.92 \min ,(p=0.046)$. The need for rescue analgesia was significantly earlier in Group II, ie at $211.09 \pm 20.74 \mathrm{~min}$, while at $256.32 \pm 24.40 \mathrm{~min}$ in group I.
\end{abstract}

Conclusion: Clonidine and butorphanol are good adjuvants to hyperbaric bupivacaine for spinal anaesthesia. Clonidine provides longer duration of sensory and motor blockade and prolonged duration of postoperative analgesia compared to butorphanol.

Key Words: Spinal Anesthesia, Clonidine, Butorphanol, Intrathecal Adjuvants, Subarachnoid Blockage.

\section{INTRODUCTION}

Neuraxial anaesthesia is a common procedure, performed during surgical proceedings of lower abdomen, pelvis and lower limbs to provide adequate surgical anaesthesia and analgesia.[1] In spinal anaesthesia, we use a small mass of drug, virtually devoid of systemic pharmacologic effect, which can produce surgical anesthesia but hemodynamic instability with unpredictable perturbations in the haemodynamic parameters as a result of sympatholysis. These effects are proportional to the level of sympathetic blockade [2]. Intrathecally administered local anaesthetics and opioids have been shown to have a synergistic analgesic effect,[3] hence requiring relatively lower dosage. Neuraxial opioids also allow prolonged analgesia in the postoperative period and faster recovery from spinal anaesthesia.[4] Anti-nociceptive synergism between local anesthetic and intrathecal 
adjuvants, opioids or $\alpha 2$ agonist, allow the reduction in the dosages of both drugs and reduce the unwanted side effects.[5]

Clonidine, a selective partial $\alpha-2$ adrenergic agonist is potent analgesic and free from opioid- related side effects of pruritus, nausea and vomiting, respiratory depression and urinary retention. It is known to increase both sensory and motor blockade of local anesthetics [6]. Butorphanol is a synthetic lipophilic opioid analgesic and used intravenously in balanced anesthetic technique but there are very few studies available in the literature on the clinical characteristics of intrathecal butorphanol.[7] Butorphanol binds to kappa receptor in the brain and spinal cord which is responsible for nociception producing analgesia devoid of mu receptors related side effect. Kappagonism also cause dysphoria at therapeutic or supertherapeutic doses and this gives butorphanol a lower potential for abuse than other opioid drugs, accounting for its easier availability in comparison to fentanyl and other potent opioids.[8] Butorphanol is a proven intravenous analgesic, effective in intra muscular route in labour analgesia, and has also been safely used in epidural anaesthesia [9-10]. In the field of infraumbilical surgeries, there is a relative paucity in literature regarding its intrathecal use, as well as its dosage in this route, especially, therefore, the present study was aimed to comparatively evaluate the clonidine and butorphanol as adjuvants to intrathecal hyperbaric bupivacaine for subarachnoid blockade during lower abdominal and lower limb surgeries. Therefore, we planned the present study to compare the efficacy and safety of intrathecal clonidine with butorphanol as adjuvants to $0.5 \%$ hyperbaric bupivacaine in patients scheduled for elective lower abdominal and lower limb surgeries.

\section{METHODS}

A double blind randomized prospective study was conducted in the Department of Anesthesia, GMC Jammu, on
60 patients of age group 18 - 58 years of both genders, undergoing various elective lower-abdominal and lower-limb surgeries under subarachnoid blockade, selected and allocated in to 2 groups of 30 each. Group-I received $30 \mu \mathrm{g}$ of clonidine $(0.2 \mathrm{ml})$ with $3.2 \mathrm{ml}$ of hyperbaric bupivacaine $0.5 \%$ (16 $\mathrm{mg}$ ) and Group-II received $200 \mu \mathrm{g}$ of butorphanol $(0.2 \mathrm{ml})$ with $3.2 \mathrm{ml}$ of hyperbaric bupivacaine $0.5 \%$ (16 $\mathrm{mg})$. Patients were randomly divided in a doubleblind manner into two equal groups of 30 patients each according to computer generated random number table. Patients were assessed in terms of time of onset of sensory and motor block, perioperative haemodynamic stability, duration of sensory and motor block, duration of analgesia and adverse effects. The drug was prepared by an anaesthesiologist who was blinded to study protocol and was not involved in further assessment of patient.

The study was approved by the Institutional Ethical Committee and written informed consent was taken from each participant. All patients were subjected to pre-anaesthetic assessment prior to enrolment. Patients were properly explained on the method of sensory and motor assessments.

Inclusion Criteria: A 11 patients of ASA (American Society of Anaesthesiologist) physical status I and II, undergoing elective lower-abdominal and lower-limb surgeries under subarachnoid blockade were included. Exclusion Criteria: Patients with history of diabetes mellitus, cardiac or pulmonary disease, hypertension, spinal deformity, severe liver disease or impaired renal functions, skin infection at site of injection, coagulation disorders, allergy to local anaesthetic, history of opioid dependence or neurological disorders and patient's refusal to technique were excluded from the study.

After arrival of patient into operation theatre, routine monitoring of non- invasive blood pressure (NIBP), heart rate (HR), electrocardiogram (ECG) and finger pulse oximetry ( $\mathrm{SpO} 2)$ was commenced, and they were preloaded with crystalloid solution at 
rate of $10 \mathrm{ml} \mathrm{kg}-1$ before initiation of subarachnoid blockade. Under all aseptic condition, lumbar puncture was performed at L3-4 inter-vertebral disc space with a 25 $\mathrm{G}$ Quincke's spinal needle in the sitting position by midline approach. After identification of correct placement by free flow of cerebral spinal fluid, $3.4 \mathrm{ml}$ of study solution was injected at rate of 0.2 $\mathrm{ml} / \mathrm{seconds}$ and then the patient was immediately turned to supine position.

Primary outcomes studied were onset and duration of sensory and motor block, duration of post-operative analgesia, sedation score, time to first rescue analgesia required and the adverse effects. The onset of sensory blockade was defined as the time from intrathecal injection to the occurrence of sensory block at dermatome level T10. The maximal cephalic level of sensory blockade and the time taken to attain, was also noted. The onset of motor blockade of the lower extremities was evaluated by observing toes movements and modified Bromage scale (0-3), at every two minutes. Definition of motor blockade according to the modified Bromage scale [11] is, 0- able to flex the whole lower limb at the hip (full motor activity), 1- able to flex the knee but unable to raise the leg at the hip, 2- able to planter flex the ankle but unable to flex the knee, 3- no movement of lower limb (no toes movements). The surgical anesthesia was considered effective when T10 dermatome was anesthetized.

Postoperatively the sensory and motor block levels were assessed at $15 \mathrm{~min}$ intervals until normal sensations returned. Duration of analgesia was taken from onset of spinal anesthesia to time of administration of first rescue analgesic, assessed using a 0-10 linear visual analogue scale (VAS) every 15 min. Rescue analgesia was given with inj. Diclofenac sodium 75 mg intramuscularly when VAS $>4$. Intraoperative hemodynamic parameters of heart rate and blood pressure were recorded at every $5 \mathrm{~min}$ in the first 30 minutes and thereafter every 15 minutes until the patient was transferred to the post anesthesia room.
Hypotension was defined as a fall in systolic blood pressure of more than $25 \%$ of base line or less than $100 \mathrm{~mm} \mathrm{Hg}$ and was treated with additional crystalloid solution and incremental dosages of mephentermine $6 \mathrm{mg}$.

Bradycardia (heart rate $<56$ beats/ min) was treated with intravenous atropine. Supplemental oxygen was administered throughout the surgery. Side effects of pruritus, nausea, vomiting, respiratory depression (respiratory rate $<10$ breaths/min or peripheral oxygen saturation $<90 \%$ ) on air or shivering was observed and managed symptomatically.

With anticipated difference between two groups taken as $15 \%$ and expected standard deviation of 20 units, minimum sample size came out to be: 56 , i.e. 28 in each group. However, the sample size was taken as 60.

The results obtained at the end of study, were tabulated as Mean \pm SD and analysed using MS Excel 2010. The demographic data for categorical variables was compared using chi-square test and statistical significance in mean difference was done by using analysis of variance (ANOVA). A $p$ value of $<0.05$ was considered significant.

\section{RESULTS}

The present study was successfully conducted on 60 enrolled patients of comparable demographic profile. There was no protocol deviation, and all were included for data analysis. Patients were cooperative with subsequent assessment of subarachnoid blockade characteristics. (Table-1)

The mean onset time of sensory block to T10 was $3.18 \pm 0.56 \mathrm{~min}$ in patients of clonidine group and $3.69 \pm 0.83$ min in patients of butorphanol group. The mean time taken for motor block up to Bromage scale 3 was $11.53 \pm 3.05 \mathrm{~min}$ in patients of clonidine group while it took $12.72 \pm 3.17 \mathrm{~min}$ in patients of butorphanol group but showed no statistical significant difference. $(\mathrm{p}=0.138)$ The duration of motor block was $294.28 \pm 33.85 \mathrm{~min}$ in patients of 
Rumani Ruku et.al. Comparative study of the effect of clonidine and butorphanol as adjuvant to intrathecal bupivacaine for post operative analgesia in lower abdominal and lower limb surgeries.

clonidine group as compared to patients of butorphanol group $245.71 \pm 30.92 \mathrm{~min}$, which was statistically significant. $(\mathrm{p}=$ $0.046)$ The need for rescue analgesia with Diclofenac sodium (VAS >4) was significantly earlier in patients of butorphanol group. (Table 2) (Figure 1)

All patients showed a decrease in heart rate and mean blood pressure, as well as systolic blood pressure, from the baseline values but the magnitude of decrease was lesser with patients of butorphanol group as compared to patients of clonidine group. No incidence of hypotension ( $\mathrm{SBP}<100 \mathrm{~mm} \mathrm{Hg}$ ) occurred in any patient, hence the vasopressor was not used. (Table 3)

Table 1: Demographic profile of patients

\begin{tabular}{|l|l|l|l|}
\hline Parameters & Group I Mean \pm SD & Group II Mean \pm SD & p- value \\
\hline Age in years & $39.66 \pm 7.12$ & $39.85 \pm 8.04$ & 0.881 \\
\hline Gender (Male: Female) & $17: 13$ & $16: 14$ & 0.456 \\
\hline BMI (Kg/m2) & $22.06 \pm 1.60$ & $21.94 \pm 1.63$ & 0.823 \\
\hline ASA I: ASAII & $19 / 11$ & $20 / 10$ & 0.648 \\
\hline Duration of surgery (in minutes) & $93.43 \pm 20.52$ & $95.36 \pm 18.46$ & 0.379 \\
\hline
\end{tabular}

Table 2: Sensory and motor blockade characteristics

\begin{tabular}{|l|l|l|l|}
\hline Parameters & Group I Mean \pm SD & Group II Mean \pm SD & P value \\
\hline Onset time of Sensory block (minutes) & $3.18 \pm 0.56$ & $3.69 \pm 0.83$ & 0.143 \\
\hline Time to two segment regression from highest sensory level (minutes) & $66.56 \pm 6.03$ & $51.49 \pm 4.82$ & $<0.013^{*}$ \\
\hline Time of onset of motor block (minutes) & $5.99 \pm 0.33$ & $6.07 \pm 0.37$ & 0.98 \\
\hline Time taken to achieve complete motor block (min) & $11.53 \pm 3.05$ & $12.72 \pm 3.17$ & 0.138 \\
\hline Duration of motor block (minutes) & $294.28 \pm 33.85$ & $245.71 \pm 30.92$ & $0.046^{*}$ \\
\hline Time to first rescue analgesia (minutes) & $256.32 \pm 24.40$ & $211.09 \pm 20.74$ & $<0.001 * *$ \\
\hline
\end{tabular}

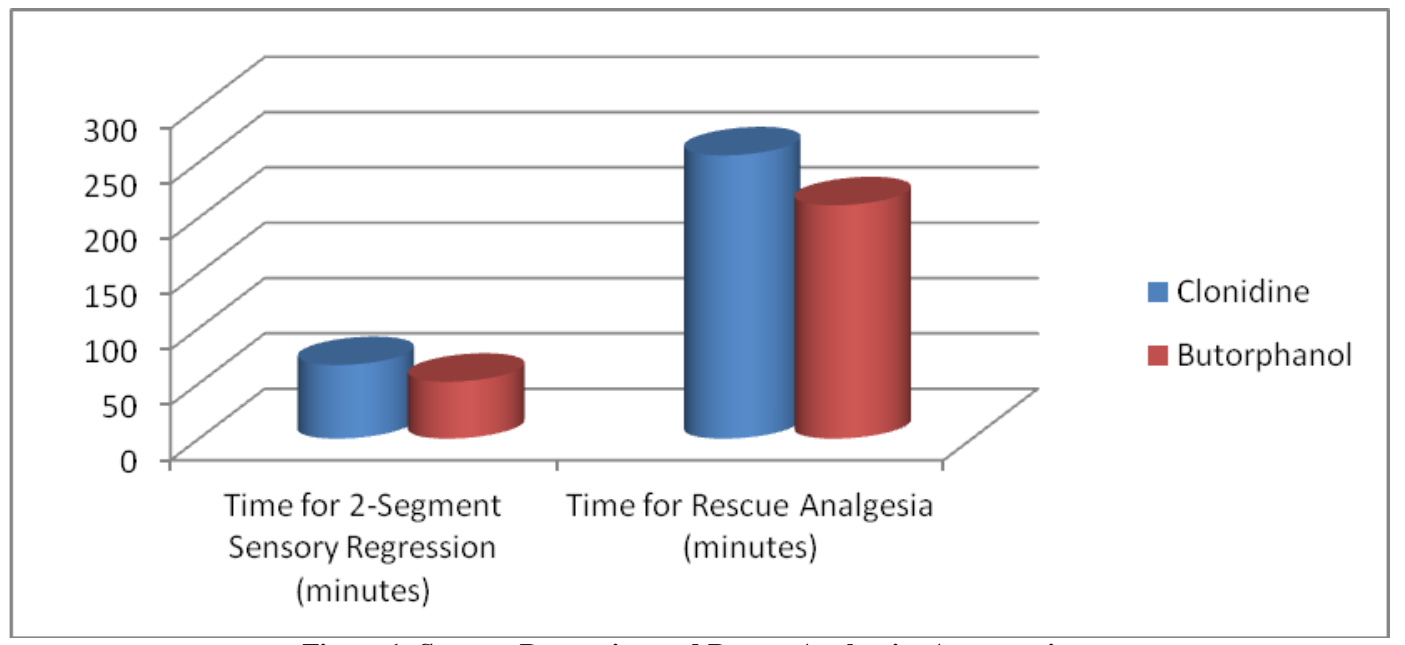

Figure 1: Sensory Regression and Rescue Analgesia: A comparison

Table 3: Hemodynamic parameters

\begin{tabular}{|c|c|c|c|c|}
\hline Parameters & Heart rate & (min) Mean \pm SD & Systolic BP (n & Hg) Mean \pm SD \\
\hline Time intervals & Group I & Group II & Group I & Group II \\
\hline Just Before SAB & $92.2 \pm 8.2$ & $99.4 \pm 7.7$ & $136.46 \pm 5.66$ & $144.59 \pm 6.82$ \\
\hline After $5 \mathrm{~min}$ & $80.3 \pm 6.6$ & $94.8 \pm 4.8$ & $120.43 \pm 5.87$ & $120.28 \pm 6.02$ \\
\hline After $10 \mathrm{~min}$ & $73.4 \pm 5.5$ & $92.1 \pm 4.5$ & $118.54 \pm 4.92$ & $120.83 \pm 5.63$ \\
\hline After $15 \mathrm{~min}$ & $64.4 \pm 4.7$ & $94.3 \pm 3.8$ & $115.42 \pm 4.96$ & $115.32 \pm 4.09$ \\
\hline After $20 \mathrm{~min}$ & $63.2 \pm 3.7$ & $89.4 \pm 3.9$ & $110.63 \pm 4.37$ & $112.03 \pm 5.80$ \\
\hline After $25 \mathrm{~min}$ & $62.8 \pm 4.1$ & $82.4 \pm 4.8$ & $108.82 \pm 3.96$ & $116.84 \pm 4.32$ \\
\hline After $30 \mathrm{~min}$ & $60.9 \pm 3.6$ & $79.5 \pm 4.5$ & $108.06 \pm 3.20$ & $107.90 \pm 4.05$ \\
\hline After $45 \mathrm{~min}$ & $62.8 \pm 3.9$ & $78.9 \pm 4.6$ & $107.31 \pm 4.57$ & $110.12 \pm 5.16$ \\
\hline After $60 \mathrm{~min}$ & $60.7 \pm 5.1$ & $75.9 \pm 3.4$ & $108.01 \pm 5.72$ & $108.85 \pm 4.23$ \\
\hline After $75 \mathrm{~min}$ & $58.9 \pm 4.5$ & $75.4 \pm 3.8$ & $104.94 \pm 7.07$ & $111.49 \pm 5.96$ \\
\hline After $90 \mathrm{~min}$ & $58.3 \pm 4.9$ & $71.9 \pm 4.2$ & $106.98 \pm 5.48$ & $117.13 \pm 8.64$ \\
\hline Postoperative & $61.0 \pm 3.3$ & $72.8 \pm 4.0$ & $108.66 \pm 6.62$ & $115.61 \pm 6.28$ \\
\hline
\end{tabular}

SAB: Subarachnoid (Spinal) Block 


\section{DISCUSSION}

The studies conducted by Gupta $\mathrm{K}$ et al., and Reddy NG et al., both of them being based on lower limb orthopaedic surgery [1,8], used a dose of $200 \mu \mathrm{gm}$ intrathecal butorphanol along with bupivacaine heavy. Similar dose was also used by Singh SN et al., in abdominal and vaginal hysterectomies under spinal anaesthesia without any significant side effects [12].

Haemodynamic parameters like mean HR, SBP and DBP were within acceptable limits. Both mean SBP and DBP were lower in the clonidine group as compared to the butorphanol group, with varied statistical significance. Haemodynamic parameters were within acceptable limits in line with the study of Reddy IR et al., [13].

Significant finding in this study was in respect of two segment regression time. It was significantly prolonged in clonidine group as compared to butorphanol group. Both these adjuvants did not cause any significant increase in onset of motor blockade. The mean time taken for motor block up to Bromage scale 3 was $11.53 \pm$ $3.05 \mathrm{~min}$ in patients of clonidine group while it took $12.72 \pm 3.17 \mathrm{~min}$ in patients of butorphanol group. The mean onset time of sensory analgesia was $3.18 \pm 0.56 \mathrm{~min}$ in patients of clonidine group and $3.69 \pm 0.83$ min in patients of butorphanol group but showed no statistically significant difference. $(\mathrm{p}=0.138)$ The duration of motor block was $294.28 \pm 33.85 \mathrm{~min}$ in patients of clonidine group as compared to patients of butorphanol group $245.71 \pm$ $30.92 \mathrm{~min}$, which was statistically significant. $(p=0.046)$ The need for rescue analgesia with Diclofenac sodium (VAS >4) was significantly earlier in patients of butorphanol group.

Most significant finding of this study was that time for first request of rescue analgesia was significantly lower in Butorphanol group compared to Clonidine group.

None of the groups had episodes of hypotension which means that even butorphanol as an adjuvant in spinal anaesthesia is quite suitable. Delayed respiratory depression is more commonly associated with poorly lipid-soluble narcotic drugs, like morphine.[14] It was suggested by Bromage PR that lipid-soluble, highly protein bound narcotic analgesics might have lesser probability to exhibit these characteristics.[15]

Our findings were similar to study conducted by Dobrydnjov et al.[16] suggesting that the dose of intrathecal clonidine does not affect the mean maximal level of sensory block. Intrathecal clonidine when combined with local anesthetic significantly potentiates the intensity and duration of motor blockade possibly due to the fact that $\alpha-2$ adrenoreceptors agonists induce cellular modification in the ventral horn of the spinal cord to facilitate the action of local anesthetic and prolongation in sensory block can be due to vasoconstrictive effect of clonidine [16]. In our study we also observed enhanced intensity and duration of motor block in patients of clonidine group as compared to butorphanol group with statistically significant difference. The intrathecal adjuvants are synergistic with local anesthetic agents to intensify the sensory block without increasing the level of sympathetic block $[17,18]$. The combination of local anaesthetic and adjuvants effectively inhibit multiple areas of neuronal excitability to provide a dose sparing effects of local anaesthetics.

A significant fall was observed in the arterial blood pressure after intrathecal clonidine administration in our study. Consistent to our findings, Saxena et al. evaluated the safest and effective lowest dose of intrathecal clonidine as adjuvant to hyperbaric bupivacaine and concluded that addition of clonidine in dose of $30 \mu \mathrm{g}$ would reduce the onset time with increase in the duration of spinal anesthesia [19]. We observed in the present study that intrathecal clonidine and butorphanol as adjuvant to $0.5 \%$ hyperbaric bupivacaine led to adequate surgical analgesia with stable 
intraoperative hemodynamic effects. No episodes of nausea and vomiting, headache, shivering and urinary retention were observed in any patients during the study.

The ventilatory frequency and peripheral oxygen saturation were comparable between the groups though patients of clonidine were well sedated as compared to patients of butorphanol group. Side effects of pruritus, shivering, nausea and vomiting did not occur. There were no transient neurological symptoms in any patients. There was no significant difference noted in respiratory rate and spo 2 in any patients during the study period. Although the patients in clonidine group were well sedated (Campbell sedation score at $1.7+$ 0.56 at $30 \mathrm{~min}$ and $1.3+0.30$ at $150 \mathrm{~min}$ ) compare to butorphanol group (Campbell sedation score at $0.5+0.40$ at $30 \mathrm{~min}$ and $0.5+0.51$ at $150 \mathrm{~min}$ ).

The inclusion of a control group in this study with a larger study group, could have further strengthened the findings. Comparative analysis with respect to gynaecological, orthopaedic and general surgery patients was also not undertaken. Future studies could cover such gap in the study.

\section{CONCLUSION}

When clonidine and butorphanol are used as an adjuvant to hyperbaric bupivacaine for spinal anaesthesia, clonidine provides longer duration of sensory and motor block compared to butorphanol. Clonidine also scored higher on sedation score and better on the time to first rescue analgesia with no respiratory depression or adverse effects. Duration of analgesia was also longer with clonidine, which delayed the time for first analgesic request compared to butorphanol. No significant haemodynamic changes or adverse effects were noted with either adjuvants. Hence, clonidine is a better adjuvant to hyperbaric bupivacaine for spinal anaesthesia with good haemodynamic stability and longer analgesia without any adverse effects.

\section{Acknowledgement: Nil}

Conflict of Interest: None

\section{Source of Funding: Nil}

Ethical Approval: Approved

\section{REFERENCES}

1. Gupta K, Jain M, Gupta PK, Agarwal S, Bhatia SK, Singh VP, et al. Clonidine versus butorphanol as adjuvant to $0.5 \%$ hyperbaric bupivacaine to enhance the onset and duration of subarachnoid blockade with postoperative analgesia during orthopaedic surgeries-A randomised study. Glob Anaesth Perioper Med. 2015;1(2):51-54.

2. Churchill Davidson HC (2003) Spinal and epidural block. In: Wylie \& Churchill Davidson- A practice of Anaesthesia, (7th Edn.), London: 608.

3. Courtney MA, Bader AM, Hartwell B, Hauch M, Grennan MJ, Datta S. Perioperative analgesia with subarachnoid sufentanil administration. Reg Anaesth. 1992;17:274-78.

4. Kuusniemi KS, Pihlajamäki KK, Pitkänen MT, Helenius HY, Kirvelä OA. The use of bupivacaine and fentanyl for spinal anaesthesia for urologic surgery. Anaesth Analg. 2000;91:1452-56.

5. Juliao MC, Lauretti GR (2000) Low-dose intrathecal clonidine combined with sufentanil as analgesic drugs in abdominal gynecological surgery. J Clin Anesth 12: 357-362.

6. Brian D Sites, Micheal Beach, Russel Biggs (2003) Intrathecal Clonidine Added to a Bupivacaine-Morphine Spinal Anesthetic Improves Postoperative Analgesia for Total Knee Arthroplasty. Anesth Analg 2003; 96 : 1083-8.

7. Kumar B, Williams A, Liddle D, Verghese $M$ (2011) Comparison of intrathecal bupivacaine-fentanyl and bupivacainebutorphanol mixtures for lower limb orthopedic procedures. Anesth Essays Res 5: 190-195.

8. Reddy NG, Manohar S, Supriya P, Himani A. Comparison of efficacy of butorphanol and fentanyl as intrathecal adjuvant to bupivacaine. Journal of Evolution of Medical and Dental Sciences. 2015;4(33): 5675-81. 
Rumani Ruku et.al. Comparative study of the effect of clonidine and butorphanol as adjuvant to intrathecal bupivacaine for post operative analgesia in lower abdominal and lower limb surgeries.

9. Yadav J, Regmi MC, Basnet P, Guddy KM, Bhattarai B, Poudel P. Butorphanol in labour analgesia. JNMA J Nepal Med Assoc. 2018;56(214):940-44. Doi: 10.31729/jnma.3905.

10. Kaur J, Bajwa SS. Comparison of epidural butorphanol and fentanyl as adjuvants in the lower abdominal surgery: A randomised clinical study. Saudi J Anaesth. 2014;8:16771.

11. Bromage PR (1978) Epidural Analgesia. Philadelphia: WB Saunders, pp: 144.

12. Singh SN, Subedi A, Prasad JN, Regmi MC. A comparative study to assess the effect of intrathecal bupivacaine with morphine or butorphanol on post-operative pain relief following abdominal and vaginal hysterectomy. Health Renaissance. 2013;11(3):246- 49.

13. Reddy IR, Aasim SA, Komravell KK. A comparative study of efficacy of anaesthesia and analgesia between intrathecal fentanyl and butorphanol with bupivacaine $0.5 \%$ heavy for lower limb orthopedic surgery: A prospective randomised study in a tertiary care teaching hospital. Int J Res Health Sci. 2018;6(2):01-08.

14. Camporesi EM, Nielsen $\mathrm{CH}$, Bromage PR, Durant PA. Ventilatory $\mathrm{CO} 2$ sensitivity after intravenous and epidural morphine in volunteers. Anaesth Analg. 1983;62:63340.

15. Bromage PR. The price of intraspinal narcotic analgesia: Basic constraints. Anaesth Analg. 1981;60(7):461-63.
16. Dobrydnjov I, Axelsson K, Thörn SE, Matthiesen P, Klockhoff H, et al. (2003) Clonidine combined with small-dose bupivacaine during spinal anesthesia for inguinal herniorrhaphy: a randomized double-blinded study. Anesth Analg 96: 1496- 1503.

17. Thakur A, Bhardwaj M, Kaur K, Dureja J, Hooda S, et al. (2013) Intrathecal clonidine as an adjuvant to hyperbaric bupivacaine in patients undergoing inguinal herniorrhaphy: A randomized double-blinded study. J Anaesthesiol Clin Pharmacol 29: 66-70.

18. Agarwal D, Chopra M, Mohta M, Sethi AK (2014) Clonidine as an adjuvant to hyperbaric bupivacaine for spinal anesthesia in elderly patients undergoing lower limb orthopedic surgeries. Saudi J Anaesth 8: 209-214.

19. Saxena H, Singh S, Ghildiyal S (2010) Low Dose Intrathecal Clonidine with Bupivacaine improves onset and duration of block with hemodynamic Stability. Internet Journal of Anesthesiology 23: 1.

How to cite this article: Rumani Ruku, Sanam Kassana. Comparative study of the effect of clonidine and butorphanol as adjuvant to intrathecal bupivacaine for post operative analgesia in lower abdominal and lower limb surgeries. International Journal of Research and Review. 2022; 9(1): 298-304. DOI: https://doi. org/10.52403/ijrr.20220135 